\title{
The impact of waist circumference on function and physical activity in older adults: longitudinal observational data from the osteoarthritis initiative
}

John A Batsis ${ }^{1,2,3^{*}}$, Alicia J Zbehlik²,3,4,5, Laura K Barre ${ }^{3,5,6}$, Todd A Mackenzie 2,3,5 $^{2}$ and Stephen J Bartels $2,3,5$

\begin{abstract}
Background: We previously demonstrated that BMI is associated with functional decline and reduced quality of life. While BMI in older adults is fraught with challenges, waist circumference (WC) is a marker of visceral adiposity that can also predict mortality. However, its association with function and quality of life in older adults is not well understood and hence we sought to examine the impact of WC on six-year outcomes.
\end{abstract}

Methods: We identified adults aged $\geq 60$ years from the longitudinal Osteoarthritis Initiative and stratified the cohort into quartiles based on WC. Our primary outcome measures of function at six year follow-up included: self-reported quality of life [Short Form-12 (SF-12)], physical function [Physical Activity Scale for the Elderly (PASE)] and disability [Late-life Disability Index (LLDI)]. Linear regression analyses predicted 6-year outcomes based on WC quartile category (lowest = referent), adjusted for age, sex, race, education, knee pain, smoking status, a modified Charlson co-morbidity index and baseline scores, where available.

Results: We identified 2,182 subjects meeting our inclusion criteria and stratified the study cohort by quartiles of WC. Mean age ranged from 67.5-68.7 years, 60-71\% were female and $80-86 \%$ were white. The highest WC quartile compared to 50-75th, 25-50th or lowest quartile, was associated with a greater number of medications (4.3, 4.0, 3.6 and $3.4[p<0.001])$, lower gait speeds $(1.23,1.27,1.32$, and $1.34 \mathrm{~m} / \mathrm{s}[\mathrm{p}<0.001])$, higher rates of knee osteoarthritis $(70.2,62.2,60.2,48.6 ; p<0.001)$, higher Charlson co-morbidity scores and greater knee pain (WOMAC scores) (all $p$ < 0.001). At follow-up, adjusted SF-12 physical function subscale and PASE scores, were lowest in the highest WC quartile as compared to the 50-75\%, 25-50\%, and lowest quartiles [(SF-12 scores: 45.5, 46.7, 47.6, and 47.9), and (PASE scores: 109.6, 128.7, 126.6, and 131.0). The LLDI limitation subscale for disability demonstrated lower scores in the high WC quartile as opposed to the referent group.

Conclusions: Elevated WC is associated with lower quality of life, a decline in physical function, and a slightly higher risk of disability over time. Intervention studies are needed to prevent functional decline in this high-risk population.

Keywords: Obesity, Waist circumference, Osteoarthritis, Disability, Physical activity, Quality of life

\footnotetext{
* Correspondence: john.batsis@gmail.com

'Section of General Internal Medicine, Dartmouth-Hitchcock Medical Center,

1 Medical Center Drive, Lebanon, NH 03756, USA

${ }^{3}$ Geisel School of Medicine at Dartmouth, 1 Rope Ferry Road, Hanover, NH 03755, USA

Full list of author information is available at the end of the article
}

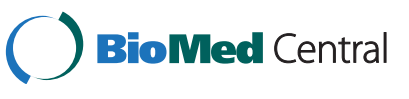

(c) 2014 Batsis et al.; licensee BioMed Central Ltd. This is an Open Access article distributed under the terms of the Creative Commons Attribution License (http://creativecommons.org/licenses/by/4.0), which permits unrestricted use, distribution, and reproduction in any medium, provided the original work is properly credited. The Creative Commons Public Domain Dedication waiver (http://creativecommons.org/publicdomain/zero/1.0/) applies to the data made available in this article, unless otherwise stated. 


\section{Background}

A number of physiological alterations occur during the aging process leading to impairments in activities of daily living that can lead to disability, institutionalization and death [1]. Osteoarthritis (OA) is an increasingly prevalent cause of functional decline and can augment the changes in body composition that occur with aging $[2,3]$. The observed decline in mobility subsequently places older adults at increased risk of falls and dependency.

Along with the rising number of older adults, the prevalence of obesity measured by body mass index continues to rise. A recent population-based survey in the United States found that 35\% of older adults are obese [4], paralleling the prevalence observed in the general population. Adiposity is associated with increased cardiometabolic risk [5], and in older adults can lead to diminished quality of life and disability [6,7]. Yet, BMI-defined obesity has poor sensitivity and specificity in identifying adiposity [8], and cannot distinguish between subcutaneous and visceral adiposity.

Waist circumference (WC) has been proposed as a relatively inexpensive measure of visceral adiposity. An elevated WC is one of five criteria that define the metabolic syndrome [9], which itself has been associated with functional decline, frailty and disability [10]. Crosssectional and longitudinal studies suggest an association of increased BMI with mobility impairments [11]. However, to our knowledge, there have been few longitudinal studies of older adults examining whether WC as a surrogate marker for visceral adiposity is associated with distal geriatric outcomes, including falls, functional decline and quality of life [11,12].

Obesity is a major risk factor for increasing severity of early degenerative changes and cartilaginous lesion progression of knee OA [13], placing older patients at risk for worsened musculoskeletal disorders and functional decline $[14,15]$. We previously demonstrated in this cohort that obesity, measured using a body mass index $(\mathrm{BMI}) \geq 30 \mathrm{~kg} / \mathrm{m}^{2}$, is associated with reduced quality of life, reduced physical activity and increased risk of disability [Batsis Submitted Public Health Nutrition] Examining the impact of central adiposity in this high-risk population allows clinicians not only to determine whether WC is a harbinger of disability but also whether it can be used as an alternative anthropometric measure. We hypothesize that in a cohort of older adults at risk for OA, that longterm quality of life, physical activity and activities of daily living are compromised in older adults with elevated WC.

\section{Methods}

The Osteoarthritis Initiative (OAI) is a multi-center, longitudinal, prospective observational study of osteoarthritis in adults. Data collection began in 2004 and is gathered at four clinical sites: Baltimore, MD, Pawtuckett,
RI, Pittsburgh, PA, and Columbus, OH. The study is funded by the National Institute of Arthritis, Musculoskeletal and Skin Diseases.

Subjects were recruited through mailings, advertisements, and community meetings. To ascertain subject eligibility, a telephone interview was conducted, and if eligible, subjects attended a screening clinic visit where additional assessments were performed. Baseline data including questionnaires, interviews, and physical assessments were collected at an enrollment clinic visit. All such visits occurred within a six-week period. Inclusion criteria included recruiting equal numbers of males and females, aged 45-79 years, and all ethnic groups were eligible for the study. Patients were excluded if they had rheumatoid arthritis, severe joint space narrowing; bilateral total knee replacements; inability to undergo an MRI; unable to provide blood samples; comorbidity preventing study participation; unlikely to be resident in the area for at least 3 years; other research participation; and unwillingness to sign an informed consent. Protocols are available online for full review. All data is freely available for download at http://www.oai.ucsf.edu/. Participants completed follow up assessments at yearly intervals and most recent study follow-up consists of six year data. For this study, baseline and six-year outcome data was used. Our local institutional review board, the Committee for the Protection of Human Subjects (CPHS\#23707), deemed the study exempt for research purposes.

\section{Study population}

The OAI study sample consisted of subjects of both sexes and of all ethnicities aged 45-79 years. All subjects were classified at baseline according to one of three subgroups: clinically significant knee osteoarthritis at risk of disease progression (progression); subjects at high risk of developing clinically significant knee OA (incident); and a control group. The progression subgroup patients complained of frequent knee symptoms or radiographic tibiofemoral knee $\mathrm{OA}$ in at least one native knee. The incident cohort was free of baseline symptomatic knee OA, but had established risk factors including the presence of heberden's nodes in both hands, increased weight; previous knee operation; previous knee injury; family history; and pain in the knee on most days of the preceding month. The control group did not have pain nor radiographic findings or risk factors.

We identified 2,585 subjects aged $\geq 60$ years and excluded subjects with missing measures of adiposity (body mass index [BMI] $(\mathrm{n}=1)$ or waist circumference [WC] $(\mathrm{n}=38)) ;$ BMI $<18.5 \mathrm{~kg} / \mathrm{m}^{2}(\mathrm{n}=4)$, and those dead at follow-up ( $n=166)$. We excluded subjects without baseline primary outcome measures (short-form $12(n=21)$ or physical activity scores $(\mathrm{n}=15))$. Subjects with incident total knee arthroplasty $(n=158)$ were also excluded to 
allow the ascertainment of the natural history of osteoarthritis in the absence of surgical intervention for the knee. The final cohort consisted of 2,182 subjects.

\section{Study measures}

All study-related data was obtained by patient selfreport or by measurements based on study protocols. Questionnaires collected data on baseline demographic, medical, social and ethnic characteristics of subjects. Age at the initial visit was considered age at baseline. We dichotomized marital status as 'married' or 'single', with the latter consisting of widow, divorced, separated or never married. Education status was classified as follows: high school (graduated or not); attended college; college graduate; graduate level. Ever smokers were considered patients who smoked $>100$ cigarettes in their life. The Western Ontario and McMaster University OA Index (WOMAC) Pain Scale was utilized to assess selfreported knee pain on a 5-point Likert scale. Pain scores ranged from $0-20$ with higher scores representing worse symptoms. Subjects with knee osteoarthritis on $\mathrm{x}$-ray were considered to have knee OA. The Charlson comorbidity index captured the degree of medical comorbidity in the cohort. Subjects were classified as having an impairment in an activity of daily living if they were unable to perform any of the following: dressing, walking, bathing, eating, getting out of bed, or toileting.

\section{Measurements}

Participant weight was measured without shoes or heavy jewelry, wearing light clothing, using a calibrated standard balance beam scale. Standing height was measured with a wall-mounted stadiometer. Waist circumference was measured at the level of the umbilicus between the lower rib and the iliac crest. BMI was calculated as weight (in kilograms) divided by height (in meters) squared. Gait speed was measured using the $20 \mathrm{~m}$ walk test, a validated measure of functional status in people with knee osteoarthritis [16]. Participants walked $20 \mathrm{~m}$ in an unobstructed corridor at their usual walking speed and were timed. Reliability and intra-class correlations ranged from 0.93 to 0.98 within and between testers in patients with moderate to severe knee OA.

\section{Outcome measures}

The Short-Form 12 (SF12) is a self-reported valid and reliable measure of a person's perceived health status [17]. The overall score accounts for $>90 \%$ of the statistical variance of the longer Short Form 36 scale [18], and is valid, reliable and easily self-administered Physical and mental component scores are assessed on a Likert scale, and overall SF-12 score combines the two assessments. Overall scores range from 0 to 100 and was administered as a take-home questionnaire.
The Physical Activity Scale for the Elderly (PASE) [19] is a measure of occupational, household, and leisure activities during a one-week period in older adults $>65$ years. This 26 -item instrument is reliable and valid, and can be administered by telephone, mail or be administered in-person. In this study, the scale was administered in person. The general population has a mean score of $103 \pm 64$.1, with greater scores indicating greater intensity of activity. However, no known minimally clinically important differences are available.

The validated Late-Life Function and Disability Instrument (LLDI) [20] focuses on functional limitations and frequency limitations. These domains parallel Nagi's disablement framework [21] on disability in communitydwelling adults. Functional limitations reflect a person's inability to perform daily activities, while frequency limitations characterize the inability to engage in social environments and major life tasks. Higher scores indicate higher levels of function and is scored on a $0-100$ scale. The scale corresponds to both the physical functioning subscale of the SF-36 and London Handicap Scale [22].

\section{Statistical analysis}

All data are represented as either means \pm standard deviation, or counts (percent) (Table 1). The cohort was stratified by waist circumference quartiles [<95.1, 95.1-103.2, 103.2-111, 111-149.3 cm] Baseline characteristics across groups were compared using chi square and one-way ANOVA tests. We used paired t-tests to compare baseline to six year follow-up data (Table 2). To determine the association of baseline WC on functional outcomes at six years we used multiple linear regression. The primary six-year outcomes of interest included SF-12, PASE score, and LLDI scoring. Our primary predictor was WC using quartiles (referent = lowest quartile). As differences may be introduced simply because categories reflect different points in the distribution, researchers often control for this effect by dividing variables into categories of equal size [23]. Three separate regression analyses were performed. We determined the mean follow-up scores for each functional outcome in each of the WC categories after adjusting for age, sex, education level, race, cohort type (incident, progression and control), Charlson co-morbidity score [24], current smoking status, and adjusting for baseline scores where available (Tables 3 and 4). As many indicator variables are associated with both obesity and our outcome measures, we adjusted in our analysis for a modified Charlson index including chronic obstructive pulmonary disease, ulcers, renal disorders, kidney transplant, rheumatoid arthritis, and cancer Tables 5 and 6 . Other variables excluded from this composite score included subjects that had not fulfilled criteria for these at baseline. Second, we stratified our cohort by age, as disability is known to 
Table 1 Baseline Characteristics of all subjects $>60$ years old $(n=2,182)$

\begin{tabular}{|c|c|c|c|c|c|c|}
\hline & & & Waist circumference & Quartile & & \\
\hline & Overall cohort & Low & $25-50 \%$ & $50-75 \%$ & $75 \%+$ & $p$-value \\
\hline & $\mathrm{N}=2,182$ & $n=553$ & $n=538$ & $n=548$ & $n=543$ & \\
\hline Age, years & $68.2 \pm 5.4$ & $67.9 \pm 5.4$ & $68.7 \pm 5.4$ & $68.5 \pm 5.4$ & $67.5 \pm 5.3$ & $<0.001$ \\
\hline Female Sex, \% & $1367(62.7)$ & $390(70.5)$ & $326(60.6)$ & $327(59.7)$ & $324(59.7)$ & $<0.001$ \\
\hline \multicolumn{7}{|l|}{ Education Status } \\
\hline$<$ High School & $432(19.8)$ & 93 ( 16.8) & $111(20.6)$ & $119(21.7)$ & $109(20.1)$ & \\
\hline Some College & $524(24.0)$ & 125 (22.6) & $134(24.9)$ & $128(23.4)$ & $137(25.2)$ & 0.23 \\
\hline College & $417(19.1)$ & $103(18.6)$ & $101(18.7)$ & $99(18.1)$ & $114(21.0$ & \\
\hline$>$ College & $809(37.1)$ & $232(42.0)$ & $192(35.7)$ & $202(36.9)$ & $183(33.7)$ & \\
\hline \multicolumn{7}{|l|}{ Yearly Income } \\
\hline$>\$ 50,000$ & $1115(53.7)$ & $301(57.0)$ & $280(55.2)$ & $285(54.7)$ & $249(47.8)$ & 0.02 \\
\hline \multicolumn{7}{|l|}{ Marital Status } \\
\hline Married & $1443(66.2)$ & $370(67.0)$ & $368(68.4)$ & $376(68.6)$ & $329(60.6)$ & 0.02 \\
\hline \multicolumn{7}{|l|}{ Race } \\
\hline White & $1792(82.2)$ & $473(85.7)$ & $434(80.7)$ & $451(82.3)$ & $434(79.9)$ & \\
\hline Black & $336(15.4)$ & $62(11.2)$ & $90(16.7)$ & $85(15.5)$ & $99(18.2)$ & 0.003 \\
\hline Asian & $53(2.4)$ & $9(1.6)$ & $8(1.5)$ & $2(0.4)$ & - & \\
\hline Charlson Score & $0.45 \pm 0.9$ & $0.31 \pm 0.73$ & $0.45 \pm 0.89$ & $0.48 \pm 0.96$ & $0.58 \pm 0.99$ & $<0.001$ \\
\hline Modified Charlson Score & $0.09 \pm 0.30$ & $0.05 \pm 0.21$ & $0.09 \pm 0.31$ & $0.12 \pm 0.23$ & $0.12 \pm 0.34$ & $<0.001$ \\
\hline WOMAC pain Right & $16.0 \pm 15.5$ & $8.4 \pm 11.9$ & $10.8 \pm 13.4$ & $12.3 \pm 14.3$ & $14.2 \pm 15.3$ & $<0.001$ \\
\hline WOMAC pain Left & $16.4 \pm 16.8$ & $7.8 \pm 12.6$ & $10.8 \pm 14.6$ & $12.0 \pm 15.3$ & $13.8 \pm 16.1$ & $<0.001$ \\
\hline Ever Smoker & $1084(50.1)$ & $258(46.8)$ & $256(48.1)$ & $289(53.1)$ & $281(52.2)$ & 0.10 \\
\hline \# Medications & $3.83 \pm 2.44$ & $3.41 \pm 2.27$ & $3.61 \pm 2.30$ & $3.99 \pm 2.49$ & $4.27 \pm 2.59$ & $<0.001$ \\
\hline Knee Osteoarthritis Present & $1315(60.3)$ & $269(48.6)$ & $324(60.2)$ & $341(62.2)$ & $381(70.2)$ & $<0.001$ \\
\hline Waist circumference, $\mathrm{cm}$ & $103.2 \pm 12.1$ & $88.4 \pm 5.9$ & $98.9 \pm 2.2$ & $106.7 \pm 2.3$ & $118.7 \pm 6.8$ & $<0.001$ \\
\hline Body Mass Index, $\mathrm{kg} / \mathrm{m}^{2}$ & $28.3 \pm 4.5$ & $24.1 \pm 2.9$ & $26.9 \pm 3.0$ & $29.2 \pm 2.9$ & $33.2 \pm 3.4$ & $<0.001$ \\
\hline \multicolumn{7}{|l|}{ Cohort Allocation } \\
\hline Incident & $1,595(73.1)$ & $453(81.9)$ & $401(74.5)$ & $397(72.5)$ & $344(63.4)$ & \\
\hline Progression & $577(26.4)$ & $96(17.4)$ & $133(24.7)$ & $150(27.4)$ & 198 (36.5) & $<0.001$ \\
\hline Control & $10(0.5)$ & $4(0.7)$ & $4(0.7)$ & $1(0.2)$ & $1(0.2)$ & \\
\hline
\end{tabular}

All values represent mean $\pm S D$, or count (\%).

P-value represents the ANOVA across all waist circumference quartiles categories.

Abbreviations: OA defined as having radiographic knee osteoarthritis on either knee or both knees, WOMAC Western Ontario and McMaster University

Arthritis Index.

Some fields may not add up to overall cohort totals due to missing values.

Modified Charlson score consisted of the following variables: chronic obstructive pulmonary disease, ulcers, renal disorders, kidney transplant, rheumatoid arthritis, and cancer.

change with advanced age. Separate analyses were performed on the 60-70 year age group and the $\geq 70$ year age group. Third, we stratified our analyses by knee osteoarthritis (present/absent). We performed inverse probability weighting to account for missing outcome data at follow-up. Adjusted means (95\% confidence intervals), or mean differences compared to the lowest WC quartile are presented. Body weight and body mass index were highly collinear and hence were not included in our modeling. We present the $\mathrm{R}^{2}$ of models using standard BMI categories and WC quartiles in Table 7.
All estimates were calculated using STATA version 12 (STATACorp, College Station, TX). A p value of $<0.05$ was considered statistically significant.

\section{Results}

Statistical differences were observed in age, although there was a higher proportion of females in the low WC quartile (Table 1). Charlson co-morbidity score and its modified version, WOMAC scores, and number of medications increased with increasing WC quartile. The proportion of subjects with knee OA increased by quartile. 
Table 2 Primary and follow-up outcome measures - unadjusted quartiles $(n=2,182)$

\begin{tabular}{|c|c|c|c|c|c|c|}
\hline & & & Waist circumference & Quartile & e & \\
\hline & Overall cohort & low & $25-50 \%$ & $50-75 \%$ & $75 \%+$ & $\mathrm{p}$-value \\
\hline \multicolumn{7}{|l|}{ SF-12 Score } \\
\hline \multicolumn{7}{|l|}{ Total } \\
\hline Baseline & $103.5 \pm 11.1$ & $106.0 \pm 9.9$ & $104.3 \pm 10.7$ & $102.3 \pm 11.6$ & $101.2 \pm 11.3$ & $<0.001$ \\
\hline Follow-up & $101.1 \pm 12.4$ & $103.9 \pm 11.3$ & $102.4 \pm 12.3$ & $99.4 \pm 12.7$ & $98.5 \pm 12.7$ & $<0.001$ \\
\hline p-value & $<0.001$ & $<0.001$ & $<0.001$ & $<0.001$ & $<0.001$ & \\
\hline \multicolumn{7}{|l|}{ Physical } \\
\hline Baseline & $48.7 \pm 8.9$ & $51.3 \pm 7.9$ & $49.4 \pm 8.7$ & $47.7 \pm 9.3$ & $46.5 \pm 9.11$ & $<0.001$ \\
\hline Follow-up & $46.4 \pm 9.7$ & $49.0 \pm 8.8$ & $47.4 \pm 9.7$ & $45.0 \pm 9.7$ & $44.0 \pm 10.0$ & $<0.001$ \\
\hline p-value & $<0.001$ & $<0.001$ & $<0.001$ & $<0.001$ & $<0.001$ & \\
\hline \multicolumn{7}{|l|}{ Mental } \\
\hline Baseline & $54.8 \pm 7.5$ & 54.8 & 54.9 & 54.6 & 54.7 & 0.90 \\
\hline Follow-up & $54.7 \pm 8.1$ & $54.9 \pm 7.5$ & $55.0 \pm 7.5$ & $54.4 \pm 8.6$ & $54.5 \pm 8.9$ & 0.63 \\
\hline p-value & 0.21 & 0.71 & 0.89 & 0.43 & 0.23 & \\
\hline \multicolumn{7}{|l|}{ PASE } \\
\hline Baseline & $136.4 \pm 67.2$ & $146.9 \pm 68.3$ & $139.4 \pm 68.2$ & $130.7 \pm 67.0$ & $128.8 \pm 63.8$ & $<0.001$ \\
\hline Follow-up & $123.7 \pm 64.7$ & $133.1 \pm 64.2$ & $125.3 \pm 63.5$ & $126.8 \pm 68.2$ & $108.4 \pm 58.7$ & $<0.001$ \\
\hline p-value & $<0.001$ & $<.001$ & $<0.001$ & 0.01 & $<0.001$ & \\
\hline \multicolumn{7}{|l|}{ LLDI } \\
\hline Frequency & $55.3 \pm 6.3$ & $56.2 \pm 6.3$ & $54.9 \pm 6.1$ & $55.0 \pm 6.4$ & $54.9 \pm 6.3$ & 0.004 \\
\hline Limitation & $80.9 \pm 15.3$ & $83.6 \pm 15.0$ & $82.3 \pm 15.2$ & $79.4 \pm 15.2$ & $78.3 \pm 15.2$ & $<0.001$ \\
\hline ADL Impairment & $172(10.6)$ & $31(7.3)$ & $36(8.9)$ & $42(10.4)$ & $63(16.6)$ & $<0.001$ \\
\hline \multicolumn{7}{|l|}{ Gait Speed } \\
\hline Baseline & $1.29 \pm 0.21$ & $1.34 \pm 0.22$ & $1.32 \pm 0.22$ & $1.27 \pm 0.20$ & $1.23 \pm 0.20$ & $<0.001$ \\
\hline Follow-up & $1.24 \pm 0.21$ & $1.30 \pm 0.21$ & $1.25 \pm 0.22$ & $1.23 \pm 0.19$ & $1.16 \pm 0.21$ & $<0.001$ \\
\hline p-value & $<0.001$ & $<0.001$ & $<0.001$ & $<0.001$ & $<0.001$ & \\
\hline
\end{tabular}

All values represent mean $\pm S D$, or count (\%).

P-value represents the difference between the excluded and included analytical cohort.

Abbreviations: ADL Activities of Daily Living, LLDI Late-life disability index, PASE Physical Activity Scale for the Elderly, SF- Short Form.

Comparing subjects included in our analysis to those excluded demonstrated that those excluded (either having a knee arthroplasty or have died) were older, less likely to be female, and have higher comorbidity scores and number of medications. They had lower SF-12 scores at baseline but no differences in PASE scores [Data not shown]. Notably there was high correlation between body mass index and waist circumference $(r=0.79)$.

The unadjusted functional outcome data are presented in Table 2. Outcomes are compared between WC groups at baseline and six years and outcomes are compared across time in each group. Overall and physical component scales of the SF-12 dropped with increasing waist circumference both at baseline and follow-up, and dropped significantly within each category between baseline and follow-up. Mental component scores did not change. There were marked parallel declines in PASE scores over time and between groups at each baseline and 6 years follow-up. LLDI scores diminished at followup and the proportion of ADL impairments increased significantly from $18.0 \%$ in the lowest WC quartile to $36.6 \%$ in the upper WC quartile.

Our multivariable regression models are presented in Table 3 (overall scores and stratified by age) and Table 4 (stratified by OA status). In examining by WC quartile, SF-12 decline appears to be age-dependent and subjects in the high WC quartile had markedly lower scores than the lowest quartile. High WC quartile subjects had significant declines in PASE scores as compared to the other categories but only in the $\geq 70$ year age group. The limitation component of the LLDI was most impacted in the $\geq 70$ year age group as compared to the low WC quartile. Lastly, gait speed was lowest in the highest WC quartile as compared to the other categories. Generally, the adjusted estimates were similar adjusting for either the Charlson score or the modified Charlson co-morbidity 
Table 3 Multivariable regression analysis of follow-up primary outcome measures $(n=2,182)$

\begin{tabular}{|c|c|c|c|c|c|}
\hline & & $\begin{array}{c}\text { Waist } \\
\text { Low WC }\end{array}$ & $\begin{array}{c}\text { Circumference } \\
25-50 \% \text { ile }\end{array}$ & $\begin{array}{c}\text { Quartile } \\
50-75 \% \text { ile }\end{array}$ & $75 \%+$ \\
\hline \multicolumn{6}{|l|}{ Short-Form 12 Score } \\
\hline \multirow[t]{6}{*}{ Overall Score } & Overall & 102.4 [101.0-103.9] & 102.7 [101.5-103.9] & 101.7 [100.7-102.8] & 100.5 [99.2-101.8] \\
\hline & $\Delta$ & --- & 0.28 & -0.69 & $-1.92(p=0.04)$ \\
\hline & $60-70$ & 102.9 [101.0-104.9] & 103.0 [101.3-104.8] & 102.5 [101.2-103.9] & 101.4 [99.8-103.1] \\
\hline & $\Delta$ & --- & 0.10 & -0.46 & -1.50 \\
\hline & $70+$ & $101.5[99.0-104.0$ & $102.1[100.3-104.0]$ & $100.3[98.5-102.1]$ & 99.1 [96.9-101.2] \\
\hline & $\Delta$ & --- & 0.62 & -1.22 & -2.45 \\
\hline \multirow[t]{6}{*}{ Physical } & Overall & $47.9[46.9-48.8]$ & $47.6[46.7-48.5]$ & $46.7[45.8-47.5]$ & $45.5[44.5-46.0]$ \\
\hline & $\Delta$ & --- & -0.26 & -1.20 & $-2.3(p=0.001)$ \\
\hline & $60-70$ & $47.8[46.6-49.1]$ & $48.7[47.5-49.8]$ & 47.0 [45.8-48.2] & $46.2[44.9-47.6]$ \\
\hline & $\Delta$ & --- & 0.83 & -0.85 & -1.61 \\
\hline & $70+$ & $47.7[46.0-49.4]$ & $46.4[45.0-47.9]$ & $46.2[44.9-47.5]$ & $44.4[42.9-46.0]$ \\
\hline & $\Delta$ & --- & -1.26 & -1.54 & $-3.26(p=0.004)$ \\
\hline \multirow[t]{6}{*}{ Mental } & Overall & $55.2(54.4-55.9)$ & $55.3(54.5-56.1)$ & $54.9(54.2-55.7)$ & $54.7(53.8-55.5)$ \\
\hline & $\Delta$ & -- & -0.39 & 0.16 & -0.40 \\
\hline & $60-70$ & $55.3(54.3-56.2)$ & $54.9(53.8-55.9)$ & $55.4(54.5-56.4)$ & $54.9(53.8-56.0)$ \\
\hline & $\Delta$ & --- & -0.39 & 0.16 & -0.40 \\
\hline & $70+$ & $54.9(53.6-56.2)$ & $55.6(54.4-56.8)$ & $54.3(53.0-55.0)$ & $54.5(53.1-55.9)$ \\
\hline & $\Delta$ & --- & 0.71 & -0.60 & -0.37 \\
\hline \multirow[t]{6}{*}{ Physical Activity Scale for Elderly } & Overall & $131.0(124.3-137.7)$ & $126.6(120.3-132.8)$ & $128.7(121.5-135.9)$ & $109.6(102.6-116.6)$ \\
\hline & $\Delta$ & --- & -4.47 & -2.34 & $-21.4(p<0.001)$ \\
\hline & $60-70$ & $137.4(127.8-146.9)$ & $136.4(12.7-145.1)$ & $136.4(126.7-146.2)$ & $119.9(111.1-128.7)$ \\
\hline & $\Delta$ & --- & -1.0 & -0.97 & $-17.5(p=0.006)$ \\
\hline & $70+$ & 117.5 (109.5-125.5) & $112.2(103.4-121.0)$ & $114.6(105.3-124.0)$ & $93.5(82.8-104.0)$ \\
\hline & $\Delta$ & --- & -5.2 & -2.83 & $-23.9(p<0.001)$ \\
\hline \multicolumn{6}{|l|}{ Late-Life Disability Index } \\
\hline \multirow[t]{6}{*}{ Frequency } & Overall & $56.0(55.3-56.7)$ & $55.6(54.9-56.2)$ & $55.9(55.2-56.5)$ & $55.1(54.4-55.8)$ \\
\hline & $\Delta$ & --- & -0.43 & -0.17 & -0.94 \\
\hline & $60-70$ & $56.7(55.8-57.6)$ & $56.1(55.1-57.0)$ & $56.1(55.2-57.8)$ & $55.7(54.8-56.6)$ \\
\hline & $\Delta$ & --- & -0.66 & -0.61 & -1.1 \\
\hline & $70+$ & $55.0(54.0-56.0)$ & $54.8(53.9-55.8)$ & $55.4(54.4-56.5)$ & $54.1(53.0-55.2)$ \\
\hline & $\Delta$ & -- & -0.20 & 0.43 & -0.90 \\
\hline \multirow[t]{6}{*}{ Limitation } & Overall & $83.3(81.6-84.9)$ & $83.2(81.6-84.8)$ & $81.8(80.3-83.3)$ & $79.2(77.5-81.0)$ \\
\hline & $\Delta$ & -- & -0.07 & -1.47 & $-4.05(p=0.001)$ \\
\hline & $60-70$ & $84.2(82.1-86.4)$ & $84.6(82.4-86.8)$ & $83.2(81.2-85.1)$ & 80.9 (78.9-82.9) \\
\hline & $\Delta$ & -- & 0.38 & -1.06 & $-3.3(p=0.026)$ \\
\hline & $70+$ & $81.8(79.3-84.4)$ & 81.1 (78.6-83.5) & $80.0(77.6-82.4)$ & $76.3(73.1-79.4)$ \\
\hline & $\Delta$ & --- & -0.77 & -1.86 & $-5.56(p=0.006)$ \\
\hline \multirow[t]{3}{*}{ Gait Speed } & Overall & $1.28(1.26-1.31)$ & $1.25(1.23-1.27)$ & $1.25(1.23-1.26)$ & $1.19(1.17-1.22)$ \\
\hline & $\Delta$ & --- & $-0.03(p=0.018)$ & $-0.04(p=0.06)$ & $-0.09(p<0.001)$ \\
\hline & $60-70$ & $1.29(1.26-1.32)$ & $1.28(1.25-1.31)$ & $1.27(1.24-1.29)$ & $1.24(1.21-1.27)$ \\
\hline
\end{tabular}


Table 3 Multivariable regression analysis of follow-up primary outcome measures $(\mathbf{n}=\mathbf{2}, \mathbf{1 8 2})$ (Continued)

\begin{tabular}{cccccc}
\hline$\Delta$ & -- & -0.02 & -0.02 & $0.05(p=0.009)$ \\
$70+$ & $1.27(1.23-1.31)$ & $1.19(1.15-1.23)$ & $1.21(1.17-1.24)$ & $1.11(1.07-1.16)$ \\
& $\Delta$ & -- & $-0.08(p=0.002)$ & $-0.06(p=0.015)$ & $-0.15(p<0.001)$ \\
\hline
\end{tabular}

Values represent mean score (95\% confidence interval) of the indicated metric adjusted for: Age, sex, education level, race, modified Charlson co-morbidity index, smoking status, knee OA, hip pain and cohort type (incident, progression or control), and baseline scoring (SF-12, PASE) where available. Values in 25-50\%, $50-75 \%$, high WC quartile columns represent difference from referent category (low WC). Models stratified by age adjust for the similar co-variates other than age. Modified Charlson score consists of the following variables: chronic obstructive pulmonary disease, ulcers, renal disorders, kidney transplant, rheumatoid arthritis, and cancer.

Table 4 Multivariable regression analysis of follow-up primary outcome measures $(\mathbf{n}=\mathbf{2 1 8 2})$

\begin{tabular}{|c|c|c|c|c|c|}
\hline & & $\begin{array}{c}\text { Waist } \\
\text { Low WC }\end{array}$ & $\begin{array}{c}\text { Circumference } \\
25-50 \% \text { WC }\end{array}$ & $\begin{array}{c}\text { Quartile } \\
50-75 \% \text { WC }\end{array}$ & $75 \%+$ \\
\hline \multicolumn{6}{|l|}{ Short-Form 12 Score } \\
\hline \multirow[t]{4}{*}{ Overall Score } & $\mathrm{OA}+$ & 100.1 [97.6-102.6] & 101.2 [99.6-102.9] & 100.4 [99.0-101.7] & 99.9 [98.3-101.4] \\
\hline & $\Delta$ & --- & 1.18 & 0.32 & -0.19 \\
\hline & $O A-$ & 105.6 [104.4-106.9] & 105.0 [103.3-106.7] & 104.3 [102.6-105.9] & 101.9 [99.7-104.0] \\
\hline & $\Delta$ & --- & -0.63 & -1.37 & $-3.77(p=0.002)$ \\
\hline \multirow[t]{4}{*}{ Physical } & $\mathrm{OA}+$ & $47.0[45.7-48.2]$ & $46.2[45.1-47.4]$ & 45.1 [43.9-46.2] & $44.5[43.2-45.7]$ \\
\hline & $\Delta$ & --- & -0.71 & $-1.90(p=0.02)$ & $-2.50(p=0.003)$ \\
\hline & $O A-$ & $50.2[49.0-51.4]$ & $49.9[48.4-51.3]$ & 49.8 [48.6-51.0] & 47.6 [45.9-49.3] \\
\hline & $\Delta$ & --- & -0.33 & -0.38 & -2.60 \\
\hline \multirow[t]{4}{*}{ Mental } & $\mathrm{OA}+$ & $54.9[53.9-55.9]$ & $55.1[54.0-56.2]$ & $55.2[54.2-56.3]$ & $55.5[54.5-56.5]$ \\
\hline & $\Delta$ & --- & 0.16 & 0.33 & 0.57 \\
\hline & $O A-$ & $55.3[54.3-56.4]$ & $55.4[54.4-56.5]$ & $54.4[53.2-55.5]$ & $53.0[51.4-54.6]$ \\
\hline & $\Delta$ & --- & 0.09 & -0.93 & $-2.21(p=0.015)$ \\
\hline \multirow[t]{4}{*}{ Physical Activity Scale for Elderly } & $\mathrm{OA}+$ & $128.2[119.3-137.2]$ & $121.0[113.7-128.3]$ & 128.5 [119.7-137.4] & $107.8[99.6-116.0]$ \\
\hline & $\Delta$ & --- & -7.3 & 0.31 & $-20.4(p<0.001)$ \\
\hline & $\mathrm{OA}$ - & $137.5[128.1-146.9]$ & $136.5[125.1-147.9]$ & $129.8[117.7-141.9]$ & $111.7[99.6-123.8]$ \\
\hline & $\Delta$ & --- & -1.0 & -7.7 & $-25.8(p=0.001)$ \\
\hline \multicolumn{6}{|l|}{ Late-Life Disability Index } \\
\hline \multirow[t]{4}{*}{ Frequency } & $\mathrm{OA}+$ & $55.7[54.8-56.6]$ & $55.4[54.6-56.3]$ & $55.1[54.4-55.9]$ & $54.9[54.1-55.7]$ \\
\hline & $\Delta$ & --- & -0.28 & -0.59 & -0.84 \\
\hline & $\mathrm{OA}-$ & $56.8[55.9-57.7]$ & $55.7[54.6-56.8]$ & $57.2[55.9-58.4]$ & $55.4[54.1-56.8]$ \\
\hline & $\Delta$ & --- & -1.10 & 0.36 & -1.38 \\
\hline \multirow[t]{4}{*}{ Limitation } & $\mathrm{OA}+$ & $82.0[79.9-84.2]$ & 81.8 [79.7-83.9] & $81.3[79.4-83.1]$ & $79.0[76.9-81.0]$ \\
\hline & $\Delta$ & --- & -0.18 & -2.37 & $-6.12(p=0.002)$ \\
\hline & $\mathrm{OA}-$ & $85.6[83.6-87.7]$ & $85.5[82.9-88.0]$ & $83.3[80.8-85.8]$ & $79.5[76.2-82.9]$ \\
\hline & $\Delta$ & --- & -0.22 & -0.79 & $-3.08(p=0.04)$ \\
\hline \multirow[t]{4}{*}{ Gait Speed } & $\mathrm{OA}+$ & 1.27 [1.24-1.30] & $1.23[1.20-1.26]$ & $1.23[1.21-1.25]$ & $1.18[1.15-1.21]$ \\
\hline & $\Delta$ & --- & $-0.04(p=0.04)$ & $-0.04(p=0.017)$ & $-0.09(p<0.01)$ \\
\hline & $\mathrm{OA}$ - & 1.32 [1.29-1.34] & $1.28[1.25-1.31]$ & $1.28[1.24-1.31]$ & $1.21[1.17-1.25]$ \\
\hline & $\Delta$ & -- & -0.03 & -0.036 & $-0.11(p<0.001)$ \\
\hline
\end{tabular}

$\Delta$ - represents the adjusted difference between each tertile of Waist Circumference (WC) and low WC.

Models were adjusted for age, sex, race, education, Charlson co-morbidity index, smoking status, and cohort type. Baseline scores were included where available. Modified Charlson score consisted of the following variables: chronic obstructive pulmonary disease, ulcers, renal disorders, kidney transplant, rheumatoid arthritis, and cancer. 
Table 5 Multivariable Regression Analysis of Follow-up Primary Outcome Measures $(n=2,182)$ - Using Charlson Co-Morbidity Index as a Co-Variate

\begin{tabular}{|c|c|c|c|c|c|}
\hline & & $\begin{array}{c}\text { Waist } \\
\text { Low WC }\end{array}$ & $\begin{array}{c}\text { Circumference } \\
25-50 \% \text { ile }\end{array}$ & $\begin{array}{c}\text { Quartile } \\
50-75 \% \text { ile }\end{array}$ & $75 \%+$ \\
\hline \multicolumn{6}{|l|}{ Short-Form 12 Score } \\
\hline \multirow[t]{6}{*}{ Overall Score } & Overall & 100.4 [98.3-102.5] & 101.8 [100.7-102.8] & $100.4[99.4-101.4]$ & 99.9 [98.8-101.0] \\
\hline & $\Delta$ & --- & 1.39 & -0.02 & -0.53 \\
\hline & $60-70$ & 99.5 [95.1-103.8] & 102.4 [101.0-103.9] & $101.5[100.2-102.7]$ & 100.9 [99.6-102.2] \\
\hline & $\Delta$ & -- & 2.96 & 2.00 & 1.43 \\
\hline & $70+$ & $100.7[98.5-102.8]$ & 100.8 [99.2-102.3] & $98.5[96.8-100.2]$ & 98.2 [96.3-100.0] \\
\hline & $\Delta$ & -- & 0.08 & -2.18 & $-2.53(p=0.05)$ \\
\hline \multirow[t]{6}{*}{ Physical } & Overall & $46.8[45.5-48.0]$ & 46.8 [46.0-47.6] & $45.6[44.8-46.5]$ & $45.2[44.3-46.0]$ \\
\hline & $\Delta$ & --- & 0.02 & -1.13 & $-1.60^{*}$ \\
\hline & $60-70$ & $45.9[43.5-48.3]$ & 47.8 [46.7-48.9] & $46.2[45.1-47.3]$ & $45.9[44.7-47.1]$ \\
\hline & $\Delta$ & -- & 1.90 & 0.27 & -0.02 \\
\hline & $70+$ & $47.2[45.7-48.7]$ & $45.4[44.1-46.7]$ & $44.8[43.5-46.1]$ & $43.8[42.4-45.1]$ \\
\hline & $\Delta$ & --- & $-1.84(p=0.05)$ & $-2.40(p=0.015)$ & $-3.47^{*}$ \\
\hline \multirow[t]{6}{*}{ Mental } & Overall & $54.6[53.7-55.4]$ & $55.1[54.4-55.8]$ & $54.5[53.8-55.2]$ & 54.5 [53.7-55.3] \\
\hline & $\Delta$ & --- & 0.53 & -0.02 & -0.06 \\
\hline & $60-70$ & $54.4[53.0-55.8]$ & 55.0 [54.1-55.9] & $55.2[54.3-56.0]$ & 54.8 [53.8-55.8] \\
\hline & $\Delta$ & --- & 0.56 & 0.79 & 0.38 \\
\hline & $70+$ & $54.5[53.4-55.6]$ & $55.2[54.2-56.2]$ & $53.5[52.3-54.6]$ & $54.4[53.2-55.6]$ \\
\hline & $\Delta$ & --- & 0.70 & -1.06 & -0.14 \\
\hline \multirow[t]{6}{*}{ Physical Activity Scale for Elderly } & Overall & $127.2[121.2-133.2]$ & 125.2 [119.8-130.6] & $127.8[121.5-134.1]$ & 110.7 [104.7-116.6] \\
\hline & $\Delta$ & --- & -2.04 & 0.55 & $-16.6^{*}$ \\
\hline & $60-70$ & $132.6[124.0-141.2]$ & 136.7 [129.2-144.2] & 136.3 [127.6-144.9] & 122.5 [114.7-130.3] \\
\hline & $\Delta$ & --- & 3.09 & 3.67 & -10.1 \\
\hline & $70+$ & 116.4 [108.9-124.0] & $108.6[101.1-116.2]$ & $113.6[105.2-122.1]$ & $93.2[84.2-102.2]$ \\
\hline & $\Delta$ & --- & -7.80 & -2.80 & $-23.2^{*}$ \\
\hline \multicolumn{6}{|l|}{ Late-Life Disability Index } \\
\hline \multirow[t]{6}{*}{ Frequency } & Overall & $55.5[54.8-56.1]$ & $55.1[54.5-55.6]$ & $55.3[54.7-55.8]$ & $55.1[54.4-55.7]$ \\
\hline & $\Delta$ & --- & -0.44 & -0.25 & -0.46 \\
\hline & $60-70$ & $56.0[55.1-56.9]$ & $55.6[54.8-56.4]$ & $55.7[54.9-56.5]$ & $55.6[54.8-56.4]$ \\
\hline & $\Delta$ & --- & & & \\
\hline & $70+$ & $54.7[53.8-55.7]$ & $54.3[53.5-55.5]$ & $54.5[53.6-55.5]$ & $54.2[53.2-55.2]$ \\
\hline & $\Delta$ & -- & -0.40 & -0.28 & -0.40 \\
\hline \multirow[t]{6}{*}{ Limitation } & Overall & $82.2[80.6-83.8]$ & $82.3[80.8-83.6]$ & $79.6[78.2-81.0](p=0.02)$ & $78.8[77.3-80.3] \quad(p=0.002)$ \\
\hline & $\Delta$ & --- & 0.05 & -2.60 & -3.4 \\
\hline & $60-70$ & $83.0[80.5-85.4]$ & 83.9 [82.0-85.8] & 81.0 [79.2-82.9] & $80.5[78.7-82.3]$ \\
\hline & $\Delta$ & --- & 0.97 & -1.91 & -2.42 \\
\hline & $70+$ & 81.3 [78.9-83.6] & $79.6[77.4-81.7]$ & $77.6[75.4-79.8]$ & $76.1[73.5-78.7]$ \\
\hline & $\Delta$ & --- & -1.70 & $-3.68(p=0.02)$ & $-5.16(p=0.03)$ \\
\hline \multirow[t]{3}{*}{ Gait Speed } & Overall & $1.25[1.22-1.29]$ & $1.24[1.22-1.26]$ & $1.23[1.22-1.25]$ & $1.19[1.17-1.21]$ \\
\hline & $\Delta$ & --- & 0.02 & -0.02 & $-0.07^{*}$ \\
\hline & $60-70$ & 1.25 [1.18-1.33] & $1.27[1.25-1.30]$ & $1.26[1.24-1.28]$ & $1.23[1.21-1.26]$ \\
\hline
\end{tabular}


Table 5 Multivariable Regression Analysis of Follow-up Primary Outcome Measures (n=2,182) - Using Charlson Co-Morbidity Index as a Co-Variate (Continued)

$\begin{array}{ccccc}\Delta & --- & 0.02 & 0.04 & -0.02 \\ \mathbf{7 0 +} & 1.23[1.20-1.26] & 1.18[1.15-1.21] & 1.19[1.16-1.22] & 1.12[1.08-1.15] \\ \Delta & --- & -0.05(p=0.019) & -0.04(p=0.05) & -0.11^{*}\end{array}$

Values represent mean score (95\% confidence interval) of the indicated metric adjusted for: Age, sex, education level, race, Charlson co-morbidity index, smoking status, knee OA, hip pain and cohort type (incident, progression or control), and baseline scoring (SF-12, PASE) where available. Values in 25-50\%, 50-75\%, high WC quartile columns represent difference from referent category (low WC). Models stratified by age adjust for the similar co-variates other than age.

Table 6 Multivariable Regression Analysis of Follow-up Primary Outcome Measures $(n=2182)$ - Using Charlson Co-Morbidity Index as a Co-Variate

\begin{tabular}{|c|c|c|c|c|c|}
\hline & & $\begin{array}{c}\text { Waist } \\
\text { Low WC }\end{array}$ & $\begin{array}{l}\text { Circumference } \\
\text { 25-50\% WC }\end{array}$ & $\begin{array}{c}\text { Quartile } \\
50-75 \% \text { WC }\end{array}$ & $75 \%+$ \\
\hline \multicolumn{6}{|l|}{ Short-Form 12 Score } \\
\hline \multirow[t]{4}{*}{ Overall Score } & $\mathrm{OA}+$ & $97.3[93.4-101.2]$ & $100.9[99.5-102.2]$ & 99.1 [97.8-100.4] & 98.9 [97.6-100.2] \\
\hline & $\Delta$ & --- & 3.53 & 1.74 & 1.60 \\
\hline & OA - & 104.1 [102.7-105.4] & 103.3 [101.7-104.8] & 102.7 [101.1-104.3] & 101.7 [99.8-103.6] \\
\hline & $\Delta$ & -- & -0.78 & -1.35 & $-2.4(p=0.03)$ \\
\hline \multirow[t]{4}{*}{ Physical } & $\mathrm{OA}+$ & $45.4[43.6-47.2]$ & $45.6[44.5-46.6]$ & $44.1[43.0-45.2]$ & $44.0[42.9-45.0]$ \\
\hline & $\Delta$ & --- & -0.29 & -0.85 & -1.74 \\
\hline & OA - & $49.2[48.0]$ & $48.9[47.6-50.2]$ & $48.3[46.9-49.7]$ & $47.4[46.0-48.9]$ \\
\hline & $\Delta$ & -- & 0.16 & -1.32 & -1.41 \\
\hline \multirow[t]{4}{*}{ Mental } & $\mathrm{OA}+$ & $54.1[52.7-55.4]$ & $55.3[54.3-56.2]$ & $54.8[53.9-55.8]$ & $54.9[53.9-55.8]$ \\
\hline & $\Delta$ & -- & 1.19 & 0.77 & 0.80 \\
\hline & OA - & $54.9[54.0-55.9]$ & $54.5[53.6-55.5]$ & $54.2[53.1-55.2]$ & $53.8[52.5-55.1]$ \\
\hline & $\Delta$ & ---- & -0.39 & -0.77 & -1.15 \\
\hline \multirow[t]{4}{*}{ Physical Activity Scale for Elderly } & $\mathrm{OA}+$ & $124.7[116.6-132.8]$ & $120.7[114.4-127.0]$ & $126.7[119.0-132.3]$ & $109.2[101.9-116.5]$ \\
\hline & $\Delta$ & -- & -3.95 & 1.99 & $-15.5(p=0.004)$ \\
\hline & OA - & $132.0[126.6-140.4]$ & $133.4[123.4-143.5]$ & $128.3[117.1-139.4]$ & $112.7[102.9-122.5]$ \\
\hline & $\Delta$ & -- & 1.42 & -3.74 & $-19.3(p=0.003)$ \\
\hline \multicolumn{6}{|l|}{ Late-Life Disability Index } \\
\hline \multirow[t]{4}{*}{ Frequency } & $\mathrm{OA}+$ & $55.1[54.2-56.0]$ & $55.0[54.3-55.7]$ & $54.7[54.1-55.4]$ & $54.6[53.9-55.4]$ \\
\hline & $\Delta$ & -- & -0.10 & -0.38 & -0.47 \\
\hline & OA - & $56.2[55.4-57.1]$ & $55.0[54.1-55.9]$ & $56.3[55.1-57.4]$ & $55.8[54.6-57.0]$ \\
\hline & $\Delta$ & -- & $-1.22(p=0.05)$ & 0.02 & -0.41 \\
\hline \multirow[t]{4}{*}{ Limitation } & $\mathrm{OA}+$ & 80.7 [78.4-83.0] & $81.3[79.5-83.1]$ & $79.3[77.6-81.0]$ & 78.2 [76.4-79.9] \\
\hline & $\Delta$ & --- & 0.60 & -1.38 & -2.55 \\
\hline & OA - & $84.0[82.0-86.0]$ & $83.9[81.5-86.2]$ & 80.5 [77.8-83.2] & 79.9 [77.1-82.7] \\
\hline & $\Delta$ & --- & -0.12 & $-3.49(p=0.04)$ & $-4.10(p=0.02)$ \\
\hline \multirow[t]{4}{*}{ Gait Speed } & $\mathrm{OA}+$ & 1.23 [1.17-1.29] & $1.22[1.19-1.24]$ & $1.22[1.20-1.24]$ & 1.17 [1.15-1.19] \\
\hline & $\Delta$ & & -0.02 & -0.01 & $-0.06(p=0.046)$ \\
\hline & OA - & $1.29[1.27-1.31]$ & 1.27 [1.24-1.30] & $1.26[1.23-1.29$ & $1.21[1.18-1.24]$ \\
\hline & $\Delta$ & -- & -0.02 & -0.03 & $-0.08^{*}$ \\
\hline
\end{tabular}

$\Delta$ - represents the adjusted difference between each tertile of Waist Circumference (WC) and low WC. Models were adjusted for age, sex, race, education, Charlson co-morbidity index, smoking status, and cohort type. Baseline scores were included where available. 
Table $7 \mathbf{R}^{2}$ of Multivariable waist circumference or body mass index modeling

\begin{tabular}{|c|c|c|c|c|c|}
\hline & & $\begin{array}{l}\mathrm{R}^{2} \text { of model with } \\
\text { Waist circumference }\end{array}$ & $\begin{array}{l}\text { Charlson index } \\
\text { Body mass index }\end{array}$ & $\begin{array}{l}\mathrm{R}^{2} \text { of model with } \\
\text { Waist circumference }\end{array}$ & $\begin{array}{c}\text { Modified charlson index } \\
\text { Body mass index }\end{array}$ \\
\hline \multicolumn{6}{|l|}{ Short-Form 12 Score } \\
\hline \multirow[t]{3}{*}{ Overall Score } & Overall & 0.4011 & 0.4054 & 0.3879 & 0.3943 \\
\hline & $60-70$ & 0.407 & 0.3894 & 0.3876 & 0.3971 \\
\hline & $70+$ & 0.41352 & 0.902 & 0.3902 & 0.3931 \\
\hline \multirow[t]{3}{*}{ Physical } & Overall & 0.3147 & 0.3187 & 0.3171 & 0.3194 \\
\hline & $60-70$ & 0.3154 & 0.3210 & 0.3104 & 0.3146 \\
\hline & $70+$ & 0.3102 & 0.3093 & 0.3274 & 0.3236 \\
\hline \multirow[t]{3}{*}{ Mental } & Overall & 0.2308 & 0.2313 & 0.2065 & 0.2094 \\
\hline & $60-70$ & 0.225 & 0.2258 & 0.2089 & 0.2126 \\
\hline & $70+$ & 0.2591 & 0.2564 & 0.2233 & 0.2226 \\
\hline Physical Activity Scale for & Overall & 0.2443 & 0.2425 & 0.2606 & 0.2576 \\
\hline \multirow[t]{2}{*}{ Elderly } & $60-70$ & 0.2132 & 0.2019 & 0.2298 & 0.2306 \\
\hline & $70+$ & 0.2284 & 0.2181 & 0.2550 & 0.2444 \\
\hline \multicolumn{6}{|l|}{ Late-Life Disability Index } \\
\hline \multirow[t]{3}{*}{ Frequency } & Overall & 0.1022 & 0.1022 & 0.0866 & 0.0881 \\
\hline & $60-70$ & 0.0967 & 0.0969 & 0.0849 & 0.0881 \\
\hline & $70+$ & 0.1006 & 0.1007 & 0.0709 & 0.0962 \\
\hline \multirow[t]{3}{*}{ Limitation } & Overall & 0.0936 & 0.0916 & 0.0912 & 0.913 \\
\hline & $60-70$ & 0.0987 & 0.0983 & 0.101 & 0.107 \\
\hline & $70+$ & 0.0754 & 0.0711 & 0.0873 & 0.0820 \\
\hline \multirow[t]{3}{*}{ Gait Speed } & Overall & 0.5377 & 0.05342 & 0.5277 & 0.5226 \\
\hline & $60-70$ & 0.5019 & 0.4998 & 0.4921 & 0.4911 \\
\hline & $70+$ & 0.5469 & 0.5382 & 0.5528 & 0.5368 \\
\hline
\end{tabular}

All models adjust for age, sex, education level, race, smoking status, knee OA, hip pain and cohort type (incident, progression or control), and baseline scoring (SF-12, PASE) where available. Standard Charlson Co-morbidity Index is accounted for in the $\mathrm{R}^{2}$ in the first two columns, while a modified Charlson Co-morbidity index accounting for Chronic Obstructive Pulmonary Disease, Peptic Ulcer Disease, Kidney Disease, Kidney Transplant, Rheumatoid Arthritis and

Polymyalgia Rheumatica.

index (Table 5). Patients with osteoarthritis generally had lower outcome measures as compared to those without OA (Table 6). However, significant differences were observed in PASE scores in both cohorts, yet only those without OA had differences compared to the low WC quartile for the limitation domain of the LLDI and gait speed. Model $\mathrm{R}^{2}$ were similar for predictor variables for both WC and BMI (Table 7).

\section{Discussion}

Older adults with high waist circumference are at risk for reduced self-reported health, physical activity scores, and disability. Our results also suggest that the presence of OA leads to markedly reduced quality of life and physical activity scores than in subjects without OA in those with elevated visceral adiposity.

Elevated waist circumference has been associated with progression of OA in both cross-sectional and longitudinal epidemiological studies $[25,26]$. Our study specifically targets the association of WC with geriatric outcomes, including disability, quality of life and physical activity that have not been adequately evaluated longitudinally. Previous findings have demonstrated inconsistent relationships between WC and functional decline in older adults $[27,28]$. Yet, our results are consistent with those [29] who demonstrated that the highest WC quartile was associated with mobility and agility disability in an older cohort at 2-year follow-up. We found robust relationships between visceral obesity with respect to degree of functional impairment elderly persons over time suggesting a compelling rationale for clinician encouragement of weight reduction targeting visceral or central obesity in older adults at risk for osteoarthritis.

We found that after stratification by OA status, physical component scores of the SF-12, PASE score, and LLDI scores were consistently lower in those with OA. Additionally, gait speed was lower in this cohort. These results are not surprising but the differences between high WC quartile and lowest appeared to be present only in those without OA. These findings suggest that 
the rate of decline between WC quartiles likely has plateaued well before the development of OA. While purely hypothesis generating, this data could suggest the importance of identifying visceral adiposity in a population at risk knee OA population. Providers should consider promote weight loss to delay physical disability in this population.

Our findings underscore the importance of visceral adiposity measured by easily obtained clinical variables as an important and easily obtained metric with significant predictive value in long-term outcomes for older adults at risk for osteoarthritis. Yet caution is warranted in interpreting our results that may underestimate the natural history of decline observed. First, our cohort was relatively young (mean was $\sim 68$ years). The onset of disability increases with increasing age [1] and hence longer follow-up may be needed to delineate the natural course of the obesity in relation to OA. Frailty and disability often are preceded by years of preserved or compensated functional decline which would not be reflected in our findings. Second, the recruitment was based on a community-based cohort who would be able to participate in this clinical trial and may not be fully representative of the general population of older adults in the community. Third, the degree of co-morbidity was quite modest in the study population, implying a healthier population. While these results provide a reasonable basis for future study of important longitudinal geriatric outcomes, the magnitude of our changes are modest and may not necessarily be clinically significant. A longer follow-up with a cohort with advanced medical and sociodemographic characteristics would be required to confirm our results in a more medically and functionally compromised population of older adults. Lastly, WC quartiles as our main predictor was in line with previous author's recommendations that it facilitates comparison of anthropometric indices between studies [23]. Incorporating $\mathrm{WC}$ as a continuous variable would require polynomial regression modeling, which would lead to challenges with interpretability of our results from a clinical standpoint.

We acknowledge a number of other limitations in our study. While the dataset was designed to observe longitudinal outcomes specific to OA, our analysis may not have coincided with the primary scope of the initial study design. Additionally, of those aged 60 or greater, we were missing data on a number of distal (six-year) outcomes. While we attempted to mitigate this issue by comparing included subjects vs. non-included subjects, and used a statistical methodology to account for such issues, our missing cohort appeared to have higher degree of comorbidity and socioeconomic status, suggesting that our results may indeed be an underestimate of the true effect observed. Importantly, our analysis differed from one published by Colbert et al. [30], who utilized the $\mathrm{OAI}$ and its four-year outcomes to assess the impact of obesity and race on gait speed and WOMAC scores [31]. Although our data was consistent with theirs, these authors did not specifically look at older adults, nor did they utilize PASE, SF-12 or LLDI as primary outcomes. Our results are at risk for possible overadjustment as well. We deliberately presented unadjusted data to demonstrate the similar trends after accounting for a priori variables that we believed could impact our estimates. We agree that certain variables including social support, depression, and physical measures could conceivably have been omitted and/or excluded in our analysis. To account for possible confounding between predictor variables in the Charlson index, we restricted our modified index to those factors likely not influenced by obesity or disability outcomes. Although the estimates were slightly different, the trends were not. Lastly, future studies should relate anthropometric with body composition measures and distal outcomes.

BMI fails to differentiate between central and peripheral fat stores [8]. These results focus solely on the impact of central adiposity on functional outcomes in older adults. We previously demonstrated in a similar population that BMI impacts quality of life and physical function [Batsis et al. Under Review, Public Health Nutrition], and this current study suggests that abdominal obesity could be a separate predictor of poor functional outcomes. Our intent in this study was not to differentiate which anthropometric measures is superior in predicting distal outcomes. We were unable to adjust for BMI in our modeling as the two variables were highly collinear. This was not surprising in this sample as study recruitment focused on those with risk factors for OA (including overweight and obese based on BMI) making the metrics more homogenous. We believe that future studies should consider not only the impact of elevated WC in normal BMI individuals, but the corollary to identify the potential contribution of each metric. Particularly, in older adults, there is a strong consensus that other simple anthropometric measures should be considered for assessment of adiposity, over and above BMI. While the $\mathrm{R}^{2}$ in our modeling did not differ greatly (Table 7), using WC may still be an important anthropometric variable to measure. Not considering WC as an alternative measure may ignore a considerable sample of subjects at otherwise risk of adverse outcomes as we previously have demonstrated [32] suggesting the need for future analyses to determine the impact of these combined metrics on outcomes.

Lastly, sarcopenia, the loss of muscle mass and quality with aging [33,34], impacts function and quality of life, and may be particularly present in subjects with $\mathrm{OA}$ [35]. WC is strongly associated with sarcopenic obesity and insulin resistance [36], both of which are related to 
functional decline, frailty and disability, and thought to be partially mediated by an increase in pro-inflammatory markers, including IL-1, IL-6, and TNF-a. Additionally, leptin levels are also associated both prevalent and incident central obesity and OA [37]. We suspect that there may be a link between the functional decline observed in the highest WC group in those with OA that may potentially be leptin-mediated. We also suspect that higher degrees of pro-inflammatory cytokines viscerally may be implicated in this phenomenon. Whether the alterations in body composition due to progressive replacement of muscle mass by fat mass, in addition to loss of skeletal muscle mass and function in the aging process may be implicated in this functional decline needs further evaluation. Future study should further examine whether leptin and changes in pro-inflammatory cytokine levels predict functional impairment longitudinally.

\section{Conclusion}

In older adults, high waist circumference is associated with worsening self-reported quality of life and physical activity scores. Clinicians should consider not only targeting this subgroup for aggressive weight management to prevent such functional decline, but also encourage strengthening exercises and increases in physical activity levels which both can improve joint pain and may improve overall walking performance.

\section{Competing interests}

The authors declare that they have no competing interests.

\section{Authors' contributions}

JAB: conception, design, analysis and data interpretation; drafting manuscript + critically revising for important intellectual content; approved final version of manuscript; agree to be accountable for all aspects of the work in ensuring that questions related to the accuracy or integrity of any part of the work are appropriately investigated and resolved. AJZ: conception, design, analysis and data interpretation; critically revising for important intellectual content; approved final version of manuscript; agree to be accountable for all aspects of the work in ensuring that questions related to the accuracy or integrity of any part of the work are appropriately investigated and resolved. LKB: conception, design, analysis and data interpretation; critically revising for important intellectual content; approved final version of manuscript; agree to be accountable for all aspects of the work in ensuring that questions related to the accuracy or integrity of any part of the work are appropriately investigated and resolved. TAM: design, analysis and data interpretation; critically revising for important intellectual content; approved final version of manuscript; agree to be accountable for all aspects of the work in ensuring that questions related to the accuracy or integrity of any part of the work are appropriately investigated and resolved. SJB: conception, design, data interpretation; critically revising for important intellectual content; approved final version of manuscript; agree to be accountable for all aspects of the work in ensuring that questions related to the accuracy or integrity of any part of the work are appropriately investigated and resolved.

\section{Acknowledgement}

Work was presented in part at the Gerontological Society of America Annual Meeting, New Orleans, LA, November 2013.

\section{Funding}

This work was supported in part from the Dartmouth Centers for Health and Aging, and the Junior Faculty Development Award from the Department of Medicine, Dartmouth-Hitchcock Medical Center.

The Osteoarthritis Initiative is a public-private partnership comprised of five contracts (N01-AR-2-2258; N01-AR-2-2259; N01-AR-2-2260; N01-AR-2-2261;

N01-AR-2-2262) funded by the National Institutes of Health, a branch of the Department of Health and Human Services, and conducted by the OAI Study Investigators. Private funding partners include Merck Research Laboratories; Novartis Pharmaceuticals Corporation, GlaxoSmithKline; and Pfizer, Inc. Private sector funding for the OAl is managed by the Foundation for the National Institutes of Health. This manuscript was prepared using an OAI public use data set and does not necessarily reflect the opinions or views of the OAI investigators, the $\mathrm{NIH}$, or the private funding partners.

\section{Author details}

${ }^{1}$ Section of General Internal Medicine, Dartmouth-Hitchcock Medical Center, 1 Medical Center Drive, Lebanon, NH 03756, USA. ${ }^{2}$ Centers for Health and Aging, 46 Centerra Parkway, Lebanon, NH 03766, USA. ${ }^{3}$ Geisel School of Medicine at Dartmouth, 1 Rope Ferry Road, Hanover, NH 03755, USA. ${ }^{4}$ Section of Rheumatology, Dartmouth-Hitchcock Medical Center, 1 Medical Center Drive, Lebanon, NH 03756, USA. ${ }^{5}$ The Dartmouth Institute, Dartmouth College, 30 Lafayette St, Lebanon NH 03766, USA. ${ }^{6}$ Currently address at Division of Nutritional Sciences, School of Human Ecology, Cornell University, Ithaca, NY 14850, USA.

Received: 27 February 2014 Accepted: 29 July 2014

Published: 9 August 2014

\section{References}

1. Dunlop DD, Hughes SL, Manheim LM: Disability in activities of daily living: patterns of change and a hierarchy of disability. Am J Public Health 1997, 87:378-383.

2. Lawrence RC, Felson DT, Helmick CG, Arnold LM, Choi H, Deyo RA, Gabriel S, Hirsch R, Hochberg MC, Hunder GG, Jordan JM, Katz JN, Kremers HM, Wolfe F, National Arthritis Data Workgroup: Estimates of the prevalence of arthritis and other rheumatic conditions in the United States. Part II Arthritis Rheum 2008, 58:26-35.

3. Suri P, Morgenroth DC, Hunter DJ: Epidemiology of osteoarthritis and associated comorbidities. PM R 2012, 4:S10-S19.

4. Ogden CL, Carroll MD, Kit BK, Flegal KM: Prevalence of obesity among adults: United States. NCHS Data Brief 2011-2012, 2013:1-8.

5. Lopez-Jimenez F, Batsis JA, Roger VL, Brekke L, Ting HH, Somers VK: Trends in 10-year predicted risk of cardiovascular disease in the United States, 1976 to 2004. Circ Cardiovasc Qual Outcomes 2009, 2:443-450.

6. Alley $D E$, Chang WW: The changing relationship of obesity and disability, 1988-2004. JAMA 2007, 298:2020-2027.

7. Chambers BA, Guo SS, Siervogel R, Hall G, Chumlea WC: Cumulative effects of cardiovascular disease risk factors on quality of life. J Nutr Health Aging 2002, 6:179-184.

8. Romero-Corral A, Somers VK, Sierra-Johnson J, Thomas RJ, Collazo-Clavell ML, Korinek J, Allison TG, Batsis JA, Sert-Kuniyoshi FH, Lopez-Jimenez F: Accuracy of body mass index in diagnosing obesity in the adult general population. Int J Obes (Lond) 2008, 32:959-966.

9. Batsis JA, Nieto-Martinez RE, Lopez-Jimenez F: Metabolic syndrome: from global epidemiology to individualized medicine. Clin Pharmacol Ther 2007, 82:509-524.

10. Koster A, Patel KV, Visser M, van Eijk JT, Kanaya AM, de Rekeneire N, Newman AB, Tylavsky FA, Kritchevsky SB, Harris TB, Health, Aging and Body Composition Study: Joint effects of adiposity and physical activity on incident mobility limitation in older adults. J Am Geriatr Soc 2008, 56:636-643.

11. Schaap LA, Koster A, Visser M: Adiposity, muscle mass, and muscle strength in relation to functional decline in older persons. Epidemiol Rev 2012.

12. Vincent HK, Vincent KR, Lamb KM: Obesity and mobility disability in the older adult. Obes Rev 2010, 11:568-579.

13. Laberge MA, Baum T, Virayavanich W, Nardo L, Nevitt MC, Lynch J, McCulloch CE, Link TM: Obesity increases the prevalence and severity of focal knee abnormalities diagnosed using 3 T MRI in middle-aged 
subjects-data from the Osteoarthritis Initiative. Skeletal Radiol 2012, 41:633-641.

14. Felson DT, Anderson JJ, Naimark A, Walker AM, Meenan RF: Obesity and knee osteoarthritis. The Framingham study. Ann Intern Med 1988, 109:18-24.

15. Slemenda C, Heilman DK, Brandt KD, Katz BP, Mazzuca SA, Braunstein EM, Byrd D: Reduced quadriceps strength relative to body weight: a risk factor for knee osteoarthritis in women? Arthritis Rheum 1998, 41:1951-1959.

16. Hergenroeder AL, Brach JS, Otto AD, Sparto PJ, Jakicic JM: The influence of body mass index on self-report and performance-based measures of physical function in adult women. Cardiopulm Phys Ther J 2011, 22:11-20.

17. Ware J, Kosinski M, Keller S: How to score the SF-12 Physical. The Mental Health Summary Scales. The Health Institute, New England Medical Center: Boston, MA; 1995

18. Ware J, Kosinski M, Bjorner J, Turner-Bowker D, Gandek B, Maruish M: User's manual for the SF-36v2 Health Survey. QualityMetric Incorporated: Lincoln, Ri; 2007.

19. Washburn RA, Smith KW, Jette AM, Janney CA: The Physical Activity Scale for the Elderly (PASE): development and evaluation. J Clin Epidemiol 1993, 46:153-162.

20. Sayers SP, Jette AM, Haley SM, Heeren TC, Guralnik JM, Fielding RA: Validation of the late-life function and disability instrument. J Am Geriatr Soc 2004, 52:1554-1559.

21. Nagi SZ: A study in the evaluation of disability and rehabilitation potential: concepts, methods, and procedures. Am J Public Health Nations Health 1964, 54:1568-1579.

22. Harwood RH, Rogers A, Dickinson E, Ebrahim S: Measuring handicap: the London handicap scale, a new outcome measure for chronic disease. Qual Health Care 1994, 3:11-16.

23. Flegal KM, Graubard BI: Estimates of excess deaths associated with body mass index and other anthropometric variables. Am J Clin Nutr 2009, 89:1213-1219.

24. Charlson ME, Pompei P, Ales KL, Mackenzie CR: A new method of classifying prognostic comorbidity in longitudinal studies: development and validation. J Chronic Dis 1987, 40:373-383.

25. Janssen I, Mark AE: Separate and combined influence of body mass index and waist circumference on arthritis and knee osteoarthritis. Int J Obes (Lond) 2006, 30:1223-1228.

26. Sanghi D, Srivastava RN, Singh A, Kumari R, Mishra R, Mishra A: The association of anthropometric measures and osteoarthritis knee in non-obese subjects: a cross sectional study. Clinics 2011, 66:275-279.

27. Chen $\mathrm{H}$, Bermudez Ol, Tucker $\mathrm{KL}$ : Waist circumference and weight change are associated with disability among elderly Hispanics. J Gerontol A Biol Sci Med Sci 2002, 57:M19-M25.

28. Visser M, Harris TB, Langlois J, Hannan MT, Roubenoff R, Felson DT, Wilson PW, Kiel DP: Body fat and skeletal muscle mass in relation to physical disability in very old men and women of the Framingham heart study. J Gerontol A Biol Sci Med Sci 1998, 53:M214-M221.

29. Guallar-Castillon P, Sagardui-Villamor J, Banegas JR, Graciani A, Fornes NS, Lopez Garcia E, Rodriguez-Artalejo F: Waist circumference as a predictor of disability among older adults. Obesity (Silver Spring) 2007, 15:233-244.

30. Colbert CJ, Almagor O, Chmiel JS, Song J, Dunlop D, Hayes KW, Sharma L: Excess body weight and four-year function outcomes: comparison of African Americans and whites in a prospective study of osteoarthritis. Arthritis Care Res (Hoboken) 2013, 65:5-14.

31. Tanamas SK, Wluka AE, Davies-Tuck M, Wang Y, Strauss BJ, Proietto J, Dixon $J B$, Jones G, Forbes A, Cicuttini FM: Association of weight gain with incident knee pain, stiffness, and functional difficulties: a longitudinal study. Arthritis Care Res (Hoboken) 2013, 65:34-43.

32. Batsis JA, Sahakyan KR, Rodriguez-Escudero JP, Bartels SJ, Somers VK, Lopez-Jimenez F: Normal weight obesity and mortality in United States subjects $>/=60$ years of age (from the Third National Health and Nutrition Examination Survey). Am J Cardiol 2013, 112:1592-1598.

33. Batsis JA, Barre LK, Mackenzie TA, Pratt SI, Lopez-Jimenez F, Bartels SJ: Variation in the prevalence of sarcopenia and sarcopenic obesity in older adults associated with different research definitions: dual-energy X-ray absorptiometry data from the National Health and Nutrition Examination Survey 1999-2004. J Am Geriatr Soc 2013, 61:974-980.

34. Cruz-Jentoft AJ, Baeyens JP, Bauer JM, Boirie Y, Cederholm T, Landi F, Martin FC, Michel JP, Rolland Y, Schneider SM, Topinková E, Vandewoude M,
Zamboni M, European Working Group on Sarcopenia in Older People: Sarcopenia: European consensus on definition and diagnosis. Age Ageing 2010, 39:412-423.

35. Lee S, Kim TN, Kim SH: Sarcopenic obesity is more closely associated with knee osteoarthritis than is nonsarcopenic obesity: a cross-sectional study. Arthritis Rheum 2012, 64:3947-3954.

36. Stenholm S, Harris TB, Rantanen T, Visser M, Kritchevsky SB, Ferrucci L: Sarcopenic obesity: definition, cause and consequences. Curr Opin Clin Nutr Metab Care 2008, 11:693-700.

37. Karvonen-Gutierrez CA, Harlow SD, Mancuso P, Jacobson J, de Leon CF M, Nan B: Association of leptin levels with radiographic knee osteoarthritis among a cohort of midlife women. Arthritis Care Res (Hoboken) 2013, 65:936-944.

doi:10.1186/1475-2891-13-81

Cite this article as: Batsis et al:: The impact of waist circumference on function and physical activity in older adults: longitudinal observational data from the osteoarthritis initiative. Nutrition Journal 2014 13:81.

\section{Submit your next manuscript to BioMed Central and take full advantage of:}

- Convenient online submission

- Thorough peer review

- No space constraints or color figure charges

- Immediate publication on acceptance

- Inclusion in PubMed, CAS, Scopus and Google Scholar

- Research which is freely available for redistribution

Submit your manuscript at www.biomedcentral.com/submit
C Biomed Central 